\title{
Design of Trimming Fixture for Composite Parts
}

\author{
Feng $\mathrm{Yu}^{1}$ \\ ${ }^{1}$ Shanghai Aircraft Design and Research Institute, Liaison Engineering Department, 201210 Shanghai, China
}

\begin{abstract}
The unique properties of advanced composite materials make it an ideal material for aircraft manufacturing. The processing research of composite materials has received extensive attention at home and abroad. The structure and shape of typical rib parts are analyzed, and a trimming fixture is designed to ensure that it can be applied to parts of different specifications, while ensuring easy use, making the fixture universal, convenient and economical.
\end{abstract}

\section{Introduction}

Advanced composite materials are used to process the aircraft's main bearing structure and secondary bearing structure, and their rigidity and strength are equivalent to or exceed that of aluminum alloy. At present, it is widely used in the manufacture of aircraft fuselage structures and the overall structure of small UAVs ${ }^{[1]}$.

Advanced composite materials came out in the 1970s, and their research and application can be roughly divided into four stages. The first stage: components with little force, including the front edge, fairing, spoiler, etc. The second stage: components with less force, including elevators, rudders, flaps, ailerons, etc. The third stage: parts with greater stress, including flat tails, vertical tails, etc., take the Boeing 777 as an example, using 9.9 tons of composite materials, accounting for $11 \%$ of the total weight of the structure. Main application parts: flat tail, vertical tail, flaps, ailerons, fuselage floor beams, cabin doors, etc. The fourth stage: the application in the main bearing structure of the wings and the fuselage. Different from the military aircraft, the civil aircraft has gone through a long period of time from the third stage to the fourth stage. On the one hand, it is to develop technology, on the other hand, it is to reduce costs so that it can compete with aluminum alloy.

After composite materials are formed, postprocessing is usually required to achieve the dimensions and tolerances we need. However, due to the characteristics of high hardness, high strength, poor thermal conductivity, and anisotropy, composite materials are prone to defects during the processing, such as delamination, tearing, cracks, and burrs, composite materials are difficult to process materials. However, due to the wide application of composite materials, the research on the post-processing technology of composite materials has attracted wide attention at home and abroad. Experts and scholars have focused on composite material cutting mechanism, tool material and structure design, special processing technology, surface quality influencing factors, etc. A lot of research work has been carried out in the field, and certain results have been achieved.

\section{Structure and shape of typical composite rib parts}

The typical rib part is composed of two parts: panel and honeycomb.

The panel part is composed of a web surface and a vertical rib part. The vertical rib part is used to connect with the upper and lower wall panels. At the same time, in order to avoid the long truss on the wall panel, the vertical rib part and the edge of the web surface are arranged with square notches. As shown in Figure 1.

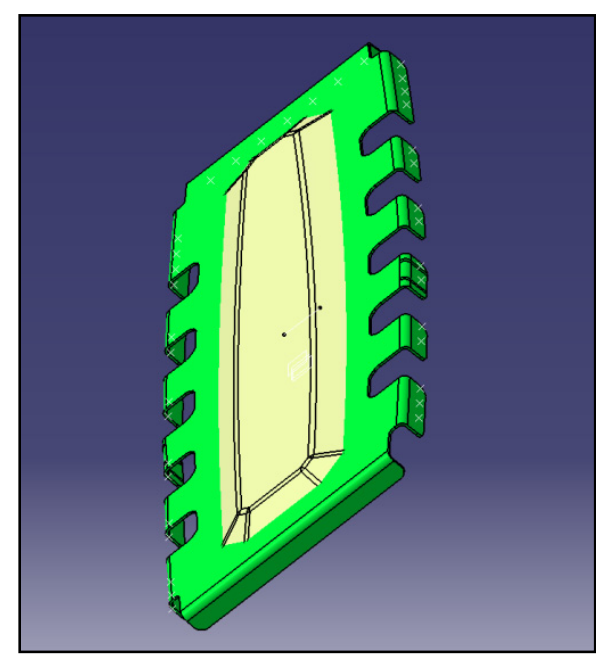

Fig. 1. Part shape.

\section{Parts requirements}

\subsection{Processing content}

The formed panel and honeycomb assembly are shown in Figure 2. The processing content is to remove the excess vertical ribs and process the square notches. 


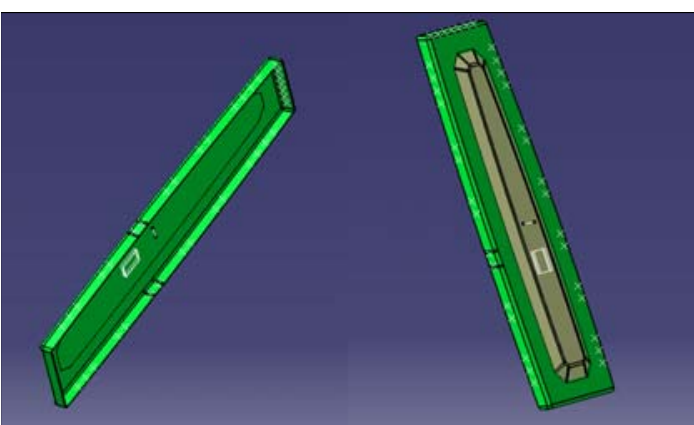

Fig. 2. The state of the part after forming.

\subsection{Processing method}

The mechanical processing of composite materials is usually divided into two methods: conventional and special. Conventional processing technology is relatively simple and the process is relatively mature, but it is difficult to process workpieces with complex shapes. The special processing method adopts the way that the tool hardly touches the workpiece or does not contact at all, and the tool wear is small. However, due to the different types of composite materials, special processing is not universal. Conventional processing methods are currently the most widely used ${ }^{[2]}$.

To obtain good performance, high safety and satisfactory results, it is necessary to establish a unique process to adapt to composite parts, and continuously optimize the processing method. Material removal rate is not the most important consideration, and the most advantageous solution should be found through economic calculations.

Wang Wenjie of Dalian University of Technology conducted experimental research on the milling and grinding of composite materials, and obtained the conclusion that the milling quality of blind grooves is not good, and the tool durability is low. He also proposed the process of "milling instead of milling". Electroplated diamond tools are the research object, and it is concluded that the "grinding instead of milling" process method can effectively improve the surface processing quality of blind grooves ${ }^{[3]}$.

Compared with the composite rib part, it meets the requirements in the above article. Analyzing the square gap of the part, it can be found that multiple changes in the processing direction will occur during the processing. Therefore, the key to the problem is how to achieve the smooth connection of the processing directions, while ensuring that the processing areas do not interfere with each other and avoiding adjacent areas Produce excessive cutting.

\section{Fixture design}

\subsection{Considerations before design}

The length of the parts is from $300 \mathrm{~mm}$ to $1000 \mathrm{~mm}$, and the width is from $200 \mathrm{~mm}$ to $500 \mathrm{~mm}$. It is processed by grinding, and special fixtures need to be designed and manufactured according to the shape of each part.
In view of the changing direction during processing, a fixing frame that can change the posture of the fixture is adopted. Since the structure of such parts is basically the same, the fixing frame can be designed as a universal one, and an index plate is used to ensure stability in each posture.

\subsection{Fixture form}

According to the changeable cutting direction, the fixture is designed as a female mold to ensure that the edge of the part is completely copied. As shown in Figure 3.

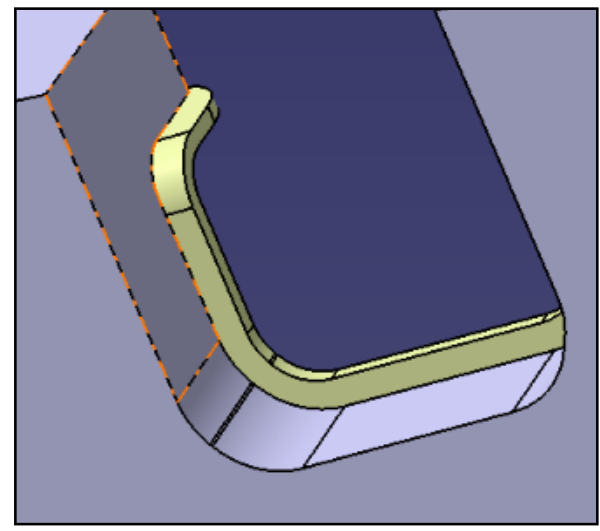

Fig. 3. Partial shape of fixture.

It should be noted that since the fixture has an opening direction, if there is not enough space for the parts on the inner opening, the parts may not be put in. Therefore, it is necessary to increase the size of the opening of the fixture to leave enough space for the shape deviation of the composite material part. As shown in Figure 4.

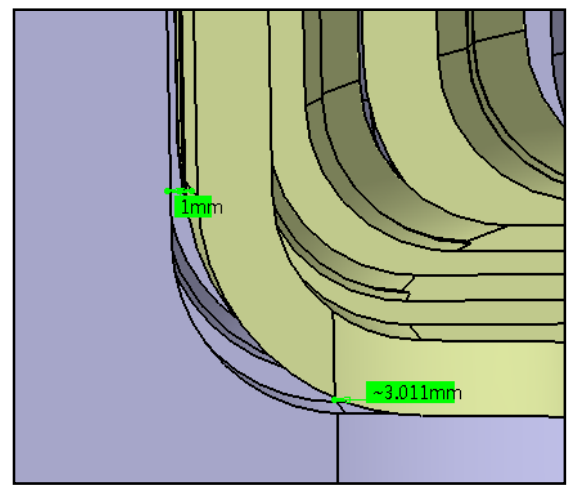

Fig. 4. Avoidance design at the opening of the fixture.

\subsection{Cutting method}

The edge of the fixture is copied from the edge of the part, and then reduced by an appropriate thickness so that the edge of the fixture is lower than the edge of the part. When machining, install the bearing on the tool side, and change the cutting amount by changing the diameter of the bearing. As shown in Figure 5. 


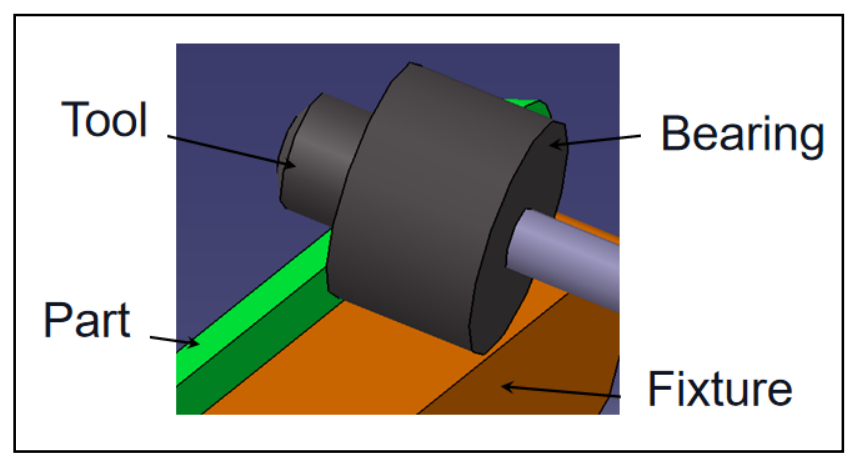

Fig. 5. Cutting method.

\subsection{Stiffness requirement}

The fixture should have sufficient stiffness, and there will be no major deformation when a force of $50 \mathrm{~N}$ is applied every $250 \mathrm{~mm}$. After being installed in the fixing frame, sufficient stability can be ensured during the cutting of parts.

\subsection{Material selection}

The panel material of this part belongs to the high temperature curing carbon fiber reinforced epoxy resin laminate, and the coefficient of linear expansion is small when heated. The length of the part is far greater than the width. The thermal expansion problem should be fully considered when selecting the master material. If the linear expansion coefficient of the part and the master does not match, the accuracy of the part's shape and size will be seriously reduced.

Among the metal materials, Invar alloy has the characteristics of small expansion coefficient, its average expansion coefficient is $1.5 \times 10^{-6}\left(/{ }^{\circ} \mathrm{C}\right)$, and the expansion coefficient reaches $1.8 \times 10^{-6}\left(/{ }^{\circ} \mathrm{C}\right)$ when the nickel content is $36 \%$, and it does not change from $-80^{\circ} \mathrm{C}$ to $100{ }^{\circ} \mathrm{C}$, and has good corrosion resistance and wear resistance $^{[4]}$. It is an ideal material for composite fixtures. The expansion coefficient of Invar alloy is closest to that of composite materials, so Invar alloy is chosen as the fixture material.

The shape of the fixture is shown in Figure 6.

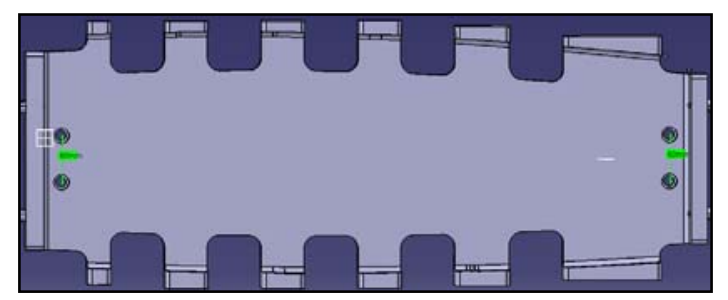

Fig. 6. Appearance of fixture.

\subsection{Deformation analysis}

Apply $50 \mathrm{~N}$ force to the fixture every $250 \mathrm{~mm}$ to ensure that the fixture does not deform greatly. The simplified diagram of force is shown in Figure 7, and the amount of deformation is shown in Figure 8.

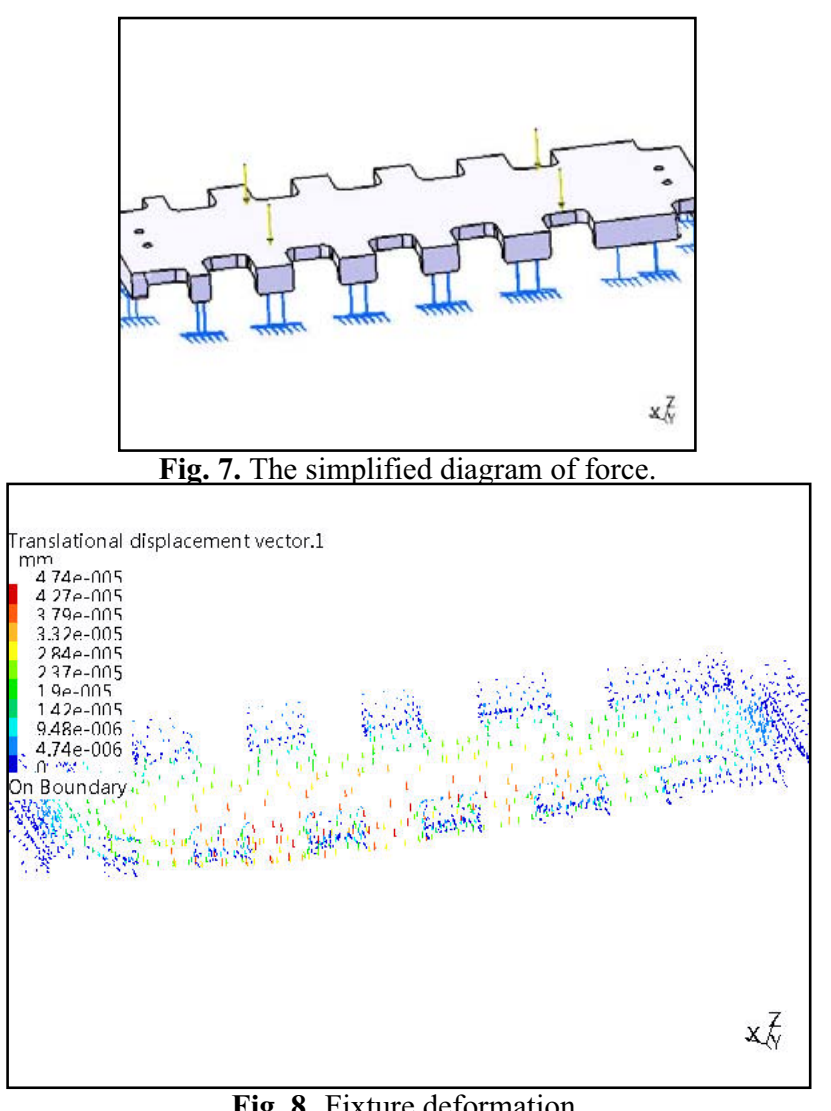

\section{Fixing frame design}

\subsection{Design requirements}

When cutting parts, the fixing frame is used to support and fix the fixture. Ensure that the fixture remains stable during cutting; at the same time, the fixture can rotate around the axis of the fixing frame and can be fixed every $45^{\circ}$ to ensure that the cutting direction is convenient for workers to operate.

After the fixing frame and the fixture are installed, there is a height difference between the trimming edge of the fixture and the standing table of the worker. This height difference should make the worker feel no discomfort during processing.

\subsection{Design points}

According to the design requirements, the combination of bearing and index plate is selected to realize the rotation of the fixing frame and the $45^{\circ}$ limit function. Due to the heavy weight of the fixture, in order to ensure convenient operation and improve safety, a worm gear reducer with self-locking function is selected to cooperate with the bearing. Use a hand wheel to turn the fixture.

The size of the fixture depends on the size of the part. The connecting holes are designed at equal intervals on the fixture, and a common mounting frame for multiple fixtures is made. In order to improve the tightness of the connection, the connecting holes on the mounting frame are arranged in a matrix on one side, and long slot- 
shaped holes are made on the other side, which not only ensures the versatility in the length direction, but also solves the problem of thermal expansion. As shown in Figure 9.

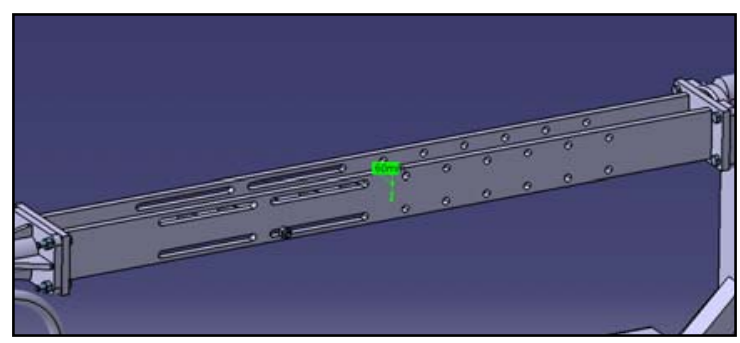

Fig. 9. Design of mounting frame.

According to Volume 6, Modeling Design and Ergonomics of the "Machine Design Manual", it can be seen that the height of the standing work plane for adult men engaged in light physical labor is $950-1100 \mathrm{~mm}^{[5]}$. According to this, the height of the fixing frame shaft is determined to be about $1000 \mathrm{~mm}$. After assembly, in each posture, the distance between the part processing position and the worker's standing table is kept within the range of $900-1100 \mathrm{~mm}$.

\subsection{Base structure of fixing frame}

The fixing frame is composed of a base, a reducer, a bearing with an index plate, a mounting frame and a hand wheel. As shown in Figure 10.

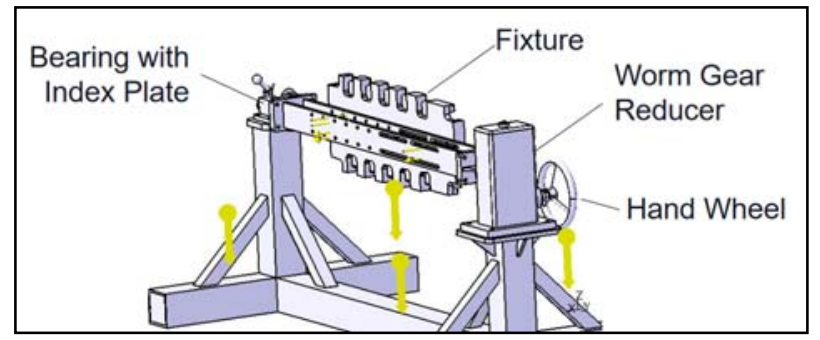

Fig. 10. The basic structure of the fixing frame.

Deformation analysis of the fixing frame, the result is shown in Figure 11.

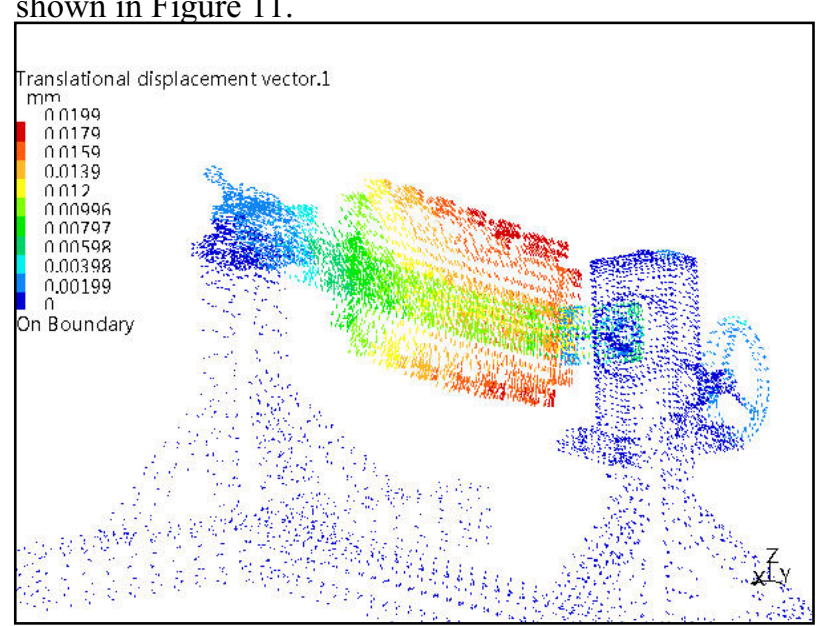

Fig. 11. The amount of deformation of the fixing frame and the fixture after the combination.

\subsection{Selection of reducer}

Generally, the maximum output power of an adult man is about $0.51 \mathrm{kw}$, which can last about $10 \mathrm{~s}$. If the duration is long, its value is much smaller, lasting $1 \mathrm{~h}$, only about $0.07-0.15 \mathrm{kw}$. For the convenience of calculation, the output power is $0.25 \mathrm{kw}$.

The rotating power of the fixture $\mathrm{P}=9550^{*} \mathrm{M}^{*} \mathrm{n}$, where $\mathrm{M}$ is the torque and $\mathrm{n}$ is the speed.

The mastering speed required by the work is relatively low. For the convenience of calculation, 1 $\mathrm{r} / \mathrm{min}$ is adopted.

It can be seen that the torque is $2.6 \times 10-5 \mathrm{~N} \cdot \mathrm{m}$

The handwheel speed is about $30 \mathrm{r} / \mathrm{min}$.

The transmission ratio is $\mathrm{i}=30 / 1=30$, and a suitable worm gear reducer is selected accordingly.

\section{Conclusion}

Through a fixing frame with multiple fixtures, the universal machining tooling design for similar parts is realized. The fixture and the fixing frame are quickly connected, the processing preparation time is short, the worker is convenient to operate, labor-saving, and easy to change the processing posture. Generally speaking, the design purpose is better achieved.

It can be seen from the design of this fixture that the development of special processing techniques and fixtures for composite rib parts can achieve highefficiency and low-cost processing compared to largescale general-purpose machine tools or machining centers.

\section{References}

1. Y.H. Su, X.J. Liu, Y.Z. Yang, Application of composite materials in aerospace, Engineering and Experiment 12, 36-38 (2008)

2. Z.N. Xiao, J.S. Zhao, Research status and analysis of composite materials processing, Proceedings of the 14th National Composite Materials Conference 2, 1099-1103 (2006)

3. W.J. Wang, Experimental research on $\mathrm{C} / \mathrm{E}$ composites grinding instead of milling, Full-text database of China's outstanding master's thesis, (2010)

4. Q.L. Li, D.C. Hu, Invar alloy characteristics and application prospects, Mechanical management and development 6, 34-35 (2007)

5. B.C. Wen, Ergonomic design criteria, Mechanical design manual 5, 56-87 (2010) 